\title{
EVALUACIÓN QUÍMICO BROMATOLÓGICA DE TRES VARIEDADES DE Arracacia xanthorrhiza "ARRACACHA"
}

\author{
Bromatological chemical evaluation of three varieties of Arracacia xanthorrhiza "arracacha”
}

Rocío Palacios ${ }^{1}$, Mariela Morales ${ }^{1}$ y Gladys C Arias ${ }^{1}$.

${ }^{1}$ Laboratorio de Bromatología, Facultad de Farmacia y Bioquímica. Universidad Nacional Mayor de San Marcos

\section{RESUMEN}

Se evaluaron las variedades amarilla, blanca y morada de Arracacia xanthorrhiza "arracacha", procedentes del distrito de Sócota, provincia de Cutervo, región de Cajamarca, proporcionadas por el Instituto Nacional de Investigación Agraria (INIA). Conocidas como r'acacha, laqachu, rakkacha, virracacha, ricacha, arracacha, racacha, arrecate, zanahoria blanca, apio criollo, sonarca, virraca, zanahoria del país y zanahoria morada, se consumen en mayor porcentaje como producto fresco. Los tallos tiernos son usados en ensaladas, cocidos o mezclados con otras raíces. Presentan un contenido de agua de 71,34 a 73,86 gramos por ciento. Las muestras secas, en gramos por ciento, presentaron: proteína total 2,10 a 2,65; ceniza 2,26 a 2,81; extracto etéreo o,91 a o,98; fibra cruda 3,25 a 3,38; carbohidratos 86,67 a 86,91; azúcares reductores totales 2,65 a 3,94 y valor calórico 363,40 a 365,78. Vitamina $C 75,75$ a 100,34 miligramos por ciento. También presenta, en muestra seca, en miligramos por ciento: calcio 116,31 a 125,36; hierro 30,26 a 41,73; fósforo 175,98 a 215,78; potasio 8,37 a 8,91; magnesio 214,12 a 261,43 y zinc 19,40 a 20,87 miligramos por ciento.

Palabras clave: Arracacia xanthorrhiza, arracacha, evaluación químico bromatológica.

\section{SUMMARY}

Were evaluated the varieties yellow, white and purple of the species Arracacia xanthorrhiza "Arracacha", , coming from Socota district of Cutervo province in department of Cajamarca, supplied for the "Instituto Nacional de Investigación Agraria" (INIA). Also are well-known like r’acacha, laqachu, rakkacha, virracacha, ricacha, arracacha, racacha, arrecate, zanahoria blanca, apio criollo, sonarca, virraca, zanahoria del país, zanahoria morada. They are consumed in greater percentage like fresh product and their stems to tier to us are used in salads or like vegetables cooked or mixed with other roots. They presents content of water of 71,34 to 73,86 grams percent. In dry sample: total protein 2,10 to 2,65; ash 2,26 to 2,81; ethereal extract o,91 to 0,98 ; raw fiber 3,25 to 3,38; carbohydrates from 86,67 to 86,91 ; total reducing sugars 2,65 to 3,94 and caloric value of 363,40 to 365,78 grams percents. Vitamin C of 75,75 to 100,34 milligrams percent. Also they presents, in dry sample: calcium from 116,31 to 125,36; iron from 30,26 to 41,73; phosphorus from 175,98 to 215,78; potassium from 8,37 to 8,91; 261,43 magnesium from 214,12 to and zinc of 19,40 to 20,87 milligrams percent.

Keywords: Arracacia xanthorrhiza, arracacha, bromatological chemical evaluation.

\section{INTRODUCCIÓN}

a arracacha (Arracacia xantorrhiza) o raíz inca es una especie nativa andina y domesticada desde tiempos muy antiguos. Los nombres tradicionales con los cuales se le conoce pertenecen al quechua usado en Perú y Colombia (r’acacha, laqachu, rakkacha) y al Aymara (r'acacha). En el Perú se le conoce como: "racacha" en Arequipa, Puno y Tacna; "virracacha" en Cuzco y Apurímac; "ricacha" en Amazonas y Cajamarca. También, con los nombres de arracacha, racacha, arrecate, zanahoria blanca, apio criollo, sonarca, virraca, afió, zanahoria del país y zanahoria morada. Se siembra entre 1000 y $2500 \mathrm{~m}$ de altitud, de preferencia en lugares con precipitaciones mayores a los $1000 \mathrm{~mm}^{3}$, observándose mayor rendimiento entre 1700 y 2500 m de altitud ${ }^{(1,2)}$.
En Colombia, Perú y Bolivia, las variedades se han reconocido por el color externo de la raíz y clasificándose en "blanca", "amarilla" y "morada".

Seencuentra distribuida en casi todoel Perú, cultivada en huertos familiares. Los centros de mayor diversidad son los distritos de Sócota y Huambos, de la provincia de Cutervo en Cajamarca, y también en Cuzco ${ }^{(3,4)}$.

El mayor porcentaje de consumo de arracacha es como producto fresco. Los tallos tiernos son usados en ensaladas o cocidos. Tradicionalmente es empleada en sopas, purés y especialmente en guisos; localmente llamados chupe (Perú), locro (Perú y Ecuador), sancocho (Colombia) y cocido (Venezuela). La preparación culinaria de las raíces es muy variada, comiéndose: cocidas, mezcladas con otras raíces, 
tubérculos y carnes; asadas, en buñuelos, en sopas y picadillos ${ }^{(5)}$.

En nuestro país hay un producto que es tradicionalmente elaborado en la comunidad de Sucse, del distrito de Sócota en la provincia de Cutervo de la región de Cajamarca, y también en Chiclayo conocido como "rallado o machacado de arracacha", el cual es una confitura obtenida a base de raíces de arracacha rallada y miel de caña de azúcar. El rallado se vende en las ferias regionales y en los mercados locales durante todo el año ${ }^{(6)}$.

En las zonas de producción forma parte de la canasta básica familiar; las raíces comestibles, muy parecidas a la yuca, son apreciadas por su agradable sabor y valor nutricional. Aunque ya se puede encontrar en algunos mercados de nuestra capital, aún pasa desapercibida, evidenciando el poco hábito de consumo de la población urbana y determinando la baja demanda del producto en los mercados. Por esto, es necesario profundizar los estudios en estos cultivos altamente promisorios y de trascendental importancia para la alimentación y desarrollo agroindustrial del país.

\section{MATERIALES Y MÉTODOS}

Se trabajó con tres variedades de Arracacia xanthorrhiza (arracacha) proporcionadas por el Instituto Nacional de Investigación Agraria (INIA), procedentes de la provincia de Cutervo, de la Región de Cajamarca (Perú). Estas tres variedades fueron: amarilla, blanca y morada.

\section{Preparación de la muestra}

Se procedió a preparar las muestras, realizando inicialmente la selección de las mismas, paraluegoserlavadas, peladas y ralladas para su homogenización. Se estabilizaron y conservaron en frascos ámbar herméticamente cerrados. En muestra fresca se determinaron, vitamina $\mathrm{C}$, humedad, azúcares totales, $\mathrm{pH}$, minerales y oligoelementos; las otras determinaciones se realizaron en muestra estabilizada.

\section{Determinaciones analíticas}

El contenido de agua, proteínas totales, extracto etéreo, cenizas y fibra cruda fueron determinados utilizando los métodos de la $\mathrm{AOAC}^{(7)}$. El factor utilizado para calcular proteína fue 6,25. Los carbohidratos fueron obtenidos por diferencia, es decir sustrayendo de 100 la suma de agua, proteína total, extracto etéreo, cenizas y fibra cruda. Los azúcares reductores directos y totales fueron determinados utilizando el método de Lane y Eynon de la AOAC ${ }^{(4)}$. Los minerales se determinaron utilizando los métodos analíticos por espectrofotometría de absorción atómica, excepto el fósforo que fue determinado por el método espectrofotométrico ${ }^{\left({ }^{8}\right)}$. La vitamina $C$ se determinó por el método de titulación con el 2,6-diclorofenolindofenol (7). La determinación de $\mathrm{pH}$ y acidez total se realizó utilizando los métodos de la AOAC ${ }^{(7)}$ y el valor calórico con el método USDA ${ }^{(9)}$.

\section{RESULTADOS}

Se presentan los resultados de la composición química, observándose que la variedad amarilla presenta valores ligeramente superiores en proteína y extracto etéreo. La variedad blanca presenta un contenido mayor de vitamina $C$, fósforo, potasio y magnesio.

\section{DISCUSIÓN}

Según Hermann y Heller, el contenido de agua en las tres variedades de arracacha fresca se encuentra en el rango de 64,12 a 81,37\%; mientras que el contenido de agua en las raíces depende de la zona de procedencia y se ve favorecido por la fertilización e irrigación excesivas ${ }^{(5)}$.

El contenido de proteína, extracto etéreo, almidón, azucares reductores totales y el valor energético en las variedades frescas, amarilla, blanca y morada, se encuentran dentro del rango mencionado por Hermann y Heller ${ }^{(5)}$.
Tabla 1. Composición químico-bromatológica de Arracacia xanthorrhiza.

\begin{tabular}{|c|c|c|c|c|c|c|}
\hline & \multicolumn{6}{|c|}{ Variedades } \\
\hline & \multicolumn{2}{|c|}{ Amarilla } & \multicolumn{2}{|c|}{ Blanca } & \multicolumn{2}{|c|}{ Morada } \\
\hline & Fresca & Seca & Fresca & Seca & Fresca & Seca \\
\hline & \multicolumn{6}{|c|}{$\mathrm{g} \%$} \\
\hline Sólidos totales & 28,66 & 100,00 & 26,37 & 100,00 & 26,14 & 100,00 \\
\hline Agua & 71,34 & 0,00 & 73,63 & 0,00 & 73,86 & 0,00 \\
\hline Proteína total $(*)$ & 0,76 & 2,65 & 0,61 & 2,34 & 0,55 & 2,10 \\
\hline Extracto etéreo & 0,28 & 0,98 & 0,24 & 0,91 & 0,24 & 0,92 \\
\hline Ceniza & 0,67 & 2,34 & 0,74 & 2,81 & 0,59 & 2,26 \\
\hline Carbohidratos & 26,95 & 86,90 & 24,78 & 86,67 & 24,76 & 86,91 \\
\hline Fibra cruda & 0,95 & 3,31 & 0,89 & 3,38 & 0,85 & 3,25 \\
\hline Azúcares reductores totales & 1,02 & 3,56 & 0,70 & 2,65 & 1,03 & 3,94 \\
\hline Almidón & 21,51 & 75,05 & 20,20 & 76,60 & 19,81 & 75,78 \\
\hline $\mathrm{pH}$ & 6,20 & - & 6,30 & - & 6,30 & - \\
\hline Vitamina $C(* *)$ & 24,78 & 86,46 & 26,46 & 100,34 & 19,80 & 75,75 \\
\hline Valor calórico $\left({ }^{* * *}\right)$ & 113,06 & 365,78 & 103,57 & 363,40 & 103,32 & 363,79 \\
\hline
\end{tabular}

${ }^{*}$ Factor de proteína $=6,25$ ** Valor expresado en $\mathrm{mg} \%{ }^{* * *}$ Valor expresado en kilocalorías 
El contenido de vitamina $\mathrm{C}$ encontrado de fue 24,$78 ; \quad 26,46$ y $19,80 \%$ en las variedades frescas amarilla, blanca y morada, respectivamente, encontrándose dentro del rango indicado por Santos ${ }^{(10)}$.

El contenido de calcio encontrado fue 34,$33 ; 30,67$ y $32,77 \quad \mathrm{mg} \%$ para las variedades frescas, amarilla, blanca y morada, respectivamente; encontrándose dentro del rango reportado por Collazos ${ }^{(9)}$ y Tapia ${ }^{(12)}$. Sin embargo, no alcanzan la media de $65 \mathrm{mg} \%$ reportado por Hermann ${ }^{(5)}$. Es muy importante tener en cuenta el aporte de calcio de la arracacha, pues supera los valores reportados para camote, oca, papa y yuca ${ }^{(9)}$.

El hierro encontrado fue 11,96; 9,52 y 7,91 mg\% en las variedades frescas amarilla, blanca y morada, respectivamente, superando ampliamente los rangos mencionados por Collazos, Montaldo y Tapia ${ }^{(9,11,12)}$.

Se observó el mayor porcentaje de zinc en la variedad amarilla con 5,98 $\mathrm{mg} \%$ seguido de 5,20 y $5,07 \mathrm{mg} \%$ en las variedades blanca y morada, respectivamente.

\section{CONCLUSIÓN}

Las variedades amarilla, blanca y morada de Arracacia xanthorrhiza "arracacha" evaluadas, presentan un contenido de agua de 71,34 a 73,86 gramos por ciento. En muestra seca, en gramos por ciento, encontramos: proteína total 2,10 a 2,65; ceniza 2,26 a 2,81; extracto etéreo o,91 a o,98; fibra cruda 3,25 a 3,38; carbohidratos 86,67 a 86,91 ; azúcares reductores totales 2,65 a 3,94 y valor calórico 363,40 a 365,78. La cantidad de Vitamina $C$ fue 75,75 a 100,34 miligramos por ciento. También presentan en muestra seca: calcio de 116,31 a 125,36; hierro 30,26 a 41,73; fósforo 175,98 a 215,78; potasio 8,37 a 8,91; magnesio 214,12 a 261,43 y zinc 19,40 a 20,87 miligramos por ciento.

\section{REFERENCIAS BIBLIOGRÁFICAS}

1. León J. Plantas alimenticias andinas. Boletín técnico 1964 (6). Instituto Interamericano de Ciencias Agrícolas de la Zona Andina. Lima, 1964.

2. National Academic of Sciences. Underexploited tropical plants with promissing value. Washington, 1975.

3. Arbizu C, Robles C. La colección de cultivos de raíces y tubérculos andinos de la Universidad de Huamanga. En el V Congreso Internacional de Sistemas Agropecuarios
Andinos. Proyecto de Investigación de Sistemas Agropecuarios Andinos. Fries, A. Lima, 1986.

4. Salas S, Seminario J, Vargas M, Seminario A. Aspectos económicos y productivos de diecisiete caseríos de Sócota, Provincia de Cutervo, involucrados en la producción de rallado de arracacha. Centro Internacional de la Papa y Universidad Nacional de Cajamarca. Lima, 1999.

5. Hermann M, Heller J. Andean Roots and tubers: ahipa, arracacha, maca and yacon. Promoting the conservation and use for underutilized and neglected crops. Institute of Plant Geneties and Crop Plant Rescarh, Gatersleben International Plant Genetic Resources Institute. Rome, 1997.

6. Seminario J. Recursos genéticos de raíces andinas: El rallado de arracacha. Universidad Nacional de Cajamarca. Cajamarca, 1998.

7. AOAC. Official Methods of Analysis of the Association of Official Analytical Chemist, 17th ed. Gaithersburg, MD. 2000.

8. Skoog D. Análisis Instrumental 4ta. Zaragoza: Ed. Editorial Mc Graw Hill, 245-26o. 1993: 245-6o.

9. Collazos C, White P, White H, Viñas E, Alvistur E, Urquieta R, et al. Composición de Alimentos. Instituto Nacional de Salud. $7^{\text {a }}$ ed. Lima, 1996.

10. Santos FF, Pereira SS. Características nutricionais de clones de mandioquinha-salsa (Arracacia xanthorrhiza Bancroft). In Tenth symposium of the International Society for Tropical Root Crops (ISTRC). Salvador de Bahia, 1994.

11. Montaldo A. Cultivo de raíces y tubérculos tropicales. Instituto Interamericano de Cooperación para la Agricultura. Servicio Editorial IICA. San José de Costa Rica, 1991.

12. Tapia M. Cultivos andinos subexplotados y su aporte en la alimentación. $2^{\text {a }}$ ed. Oficina Regional de la FAO para América Latina y el Caribe. Santiago, 1997.

Manuscrito recibido el: 22/o8/2011

Aceptado para su publicación el: 13/12/2011

\section{Correspondencia:}

Nombre: Gladys Constanza Arias Arroyo

Dirección: Av. Javier Prado Este - № 146o - Urb.

Corpac - San Isidro - Lima

e-mail: ariasarroyo@gmail.com 